European Symposium on Computer Aided Process Engineering - 15

L. Puigjaner and A. Espuña (Editors)

(C) 2005 Elsevier Science B.V. All rights reserved.

\title{
CAPE-OPEN Consultancy Scheme
}

\author{
Michel E. Pons \\ CAPE-OPEN Laboratories Network \\ $1 \& 4$ avenue de Bois-Préau, 92000 Rueil-Malmaison Cedex, France
}

\begin{abstract}
CAPE-OPEN (CO) is an industry standards on interfaces between pieces of software commonly used in process engineering. CAPE-OPEN standards is supported, enhanced and maintained by the CAPE-OPEN Laboratories Network (CO-LaN), a non-for-profit organization. CAPE-OPEN technology widens the application range of any CAPE piece of software and a number of software providers in the CAPE domain are eager to bring their software to CAPE-OPEN compliance. From the feedback CO-LaN received, CAPE-OPEN still remains a technology difficult to master quickly despite the efforts developed by CO-LaN so far. In order to overcome this difficulty, CO-LaN devised the CAPE-OPEN Consultancy Scheme where software developers received free consultancy support provided by CAPE-OPEN experts. The objective of this scheme is to catalyse faster development of $\mathrm{CO}$ compatible components. The first consultancy action proved the usefulness of the CAPE-OPEN Consultancy Scheme.
\end{abstract}

Keywords: CAPE-OPEN, CO-LaN, standard, consultancy

\section{Introduction}

Process industry companies must use a collection of software to perform Computer Aided Process Engineering (CAPE) tasks since not a single piece of software is capable to cover all end-user needs. However data and methods consistency is not often maintained between the different tools to be used. Adequate communication and integration between software tools are needed but the market for process simulation has been for many years one of incompatible proprietary products.

CAPE-OPEN and Global CAPE-OPEN projects (funded by the European Commission) aimed at overcoming these deficiencies by developing open and flexible standardized interfaces. CAPE-OPEN standards v1.0 was published at the end of the Global CAPEPEN project and subsequently polished by the CO-LaN (2003). CAPE-OPEN facilitates plug and play in CAPE tools, meaning that the same software component (unit operation module, thermodynamic property package, numerical solver) can be used in a number of process modelling environments without having to change any single line of code. Development costs for specialized CAPE tools are consequently reduced while the market for each software component is immediately expanded. There is no need anymore to develop specific interfaces with each other CAPE tool. This facility motivates software developers to migrate their product to CAPE-OPEN compliance in order to retain and achieve market competitiveness. 
On the usage side, CAPE-OPEN compliant software tools are providing end-users with an increased capability to choose and use the best tools available for the process engineering objective they have to meet. Including third party software tools in current process modelling environments is made possible, bringing an added flexibility, a larger choice, consequently an improved work process and better results while even reducing cost of process simulation work. Consequently CAPE-OPEN is a technology to widen the application range of any CAPE piece of software. The market leaders in CAPE, a number of niche software providers, as listed by Pons (2003), have adopted CAPEOPEN, ensuring a widespread usage of the technology. For example Aspen Technology Inc. recently announced at AspenWorld 2004 the release of AspenONE, an Enterprise Operation Management tool that comprises an open simulation environment that will implement interface and communication industry standards such as CAPE-OPEN.

The CAPE-OPEN Laboratories Network (CO-LaN) is the not-for-profit organization set up to maintain, support and extend the $\mathrm{CO}$ standard. Motives and organization of the CO-LaN are described by Belaud et al. (2003). CO-LaN members are process simulation end-user organizations (full members) as well as software vendors and academic institutions (associate members). Special Interest Groups (SIGs) have been formed within CO-LaN to target resources to specific issues. SIGs are gathering representatives from full and associate CO-LaN members. One such SIG is focussed on interoperability and the CAPE-OPEN Consultancy Scheme has been devised within that SIG. Representatives from BP, TOTAL, IFP, AspenTech, Process Systems Enterprise and SimSci-ESSCOR are actively involved in the Interoperability SIG.

\section{Problem definition}

The CAPE-OPEN standards rely on modern software architectures made of software components supporting communication interfaces. Since the $\mathrm{CO}$ standards are a recent development, lots of in-house, academic or commercial CAPE software do not comply with the $\mathrm{CO}$ standards yet.

Which migration strategies, e.g. by wrapping, source-code reuse or other alternatives, are feasible depends heavily on the software system and its maintenance state, but of course also on the resources and further goals related to the migration project. For example many of these systems are monolithic applications without object-oriented interfaces and are written in FORTRAN. In such a case any integration with other software is difficult and a direct integration into a component based framework like CAPE-OPEN is almost impossible. However, wrapping may be more easily achieved.

Choosing which strategy to pursue and then implementing it requests a thorough understanding of the CAPE-OPEN standards. Information on CAPE-OPEN is widely and freely accessible through the CO-LaN website. CO Tour days have been organized throughout Europe to disseminate about CAPE-OPEN. The CO Update electronic newsletter is keeping the CAPE community aware of CAPE-OPEN. More recently a CAPE-OPEN workshop was organized (August 23-24, 2004) in Cincinnatti, $\mathrm{OH}$ and sponsored by the U.S. Environmental Protection Agency to promote and help understanding of CAPE-OPEN. Despite these efforts, and according to the feedback CO-LaN received from interviewing a number of organizations for a survey it conducted on $\mathrm{CO}$ implementation (see CO-LaN website for detailed survey results), 
CAPE-OPEN remains a technology difficult to master quickly. This difficulty slows down the adoption process especially in small-size organisations. A more technical approach to dissemination and support of CAPE-OPEN seemed needed.

Already a number of actions are taking place to ease up the learning curve, such as development of wizards. A CAPE-OPEN Unit Operation wizard, developed by AspenTech for the Global CAPE-OPEN project, is available for download from the CO-LaN website (www.colan.org). It enables someone to quickly develop a Visual Basic wrapper for encapsulating a unit operation model.

Once a software component aiming at $\mathrm{CO}$ compliance is ready, it is advisable to test its compliance with the $\mathrm{CO}$ Tester Suite, freely available for download from the CO-LaN website. For example the part of the CO Tester Suite aiming at the Thermodynamic interface specification in version 1.1 is described in Benqlilou et al. (2004). Such a test provides insight on how a software component behaves within a PME.

To complement these software resources, $\mathrm{CO}-\mathrm{LaN}$ is currently considering developing a $\mathrm{CO}$ logger to log automatically any communication taking place through the $\mathrm{CO}$ interfaces. However it was felt that additional means to help developers were necessary.

\section{CAPE-OPEN Consultancy Scheme}

The CAPE-OPEN Consultancy Scheme has been initiated, proposed, approved, implemented and put in motion by the CO-LaN organization, under the drive from its Interoperability Special Interest Group.

\subsection{Initiation}

The Interoperability SIG pursues the following objectives, as defined in its charter:

- To categorise, document $\&$ publicise the level of interoperability testing that has been performed on the $\mathrm{CO}$ components that are included in the CO-LaN database.

- To provide materials and advice for carrying out interoperability testing, as well as actual testing in some cases.

- To promote the production of functional CAPE-OPEN components that interoperate successfully with the main commercial Process Modelling Environments.

Under the second objective listed here, the Interoperability SIG has been attentive to the difficulties met by several niche software providers when migrating their products to CAPE-OPEN compliance. Consequently, the Interoperability SIG proposed a Consultancy Scheme to the CO-LaN Management Board. The Board gave approval for the scheme on October 1, 2003.

\subsection{Scheme details}

Under the Consultancy Scheme a software component developer wishing to create a CO compliant Process Modelling Environment (PME) or Process Modelling Component (PMC) may receive free support from a CO-LaN approved CO Consultant. The software component developer fills a Consultancy Request Form (available from www.colan.org) and forwards it to the CO-LaN Management Board or to a specific full CO-LaN member. The following preconditions apply:

- The developer should show CAPE-OPEN tester results if such a tester exists for the implemented interfaces. 
- Once developed, the to-be-migrated piece of software must be accessible to all full members of CO-LaN on a commercial basis.

CO-LaN is funding up to two person days of CAPE-OPEN consultancy for each development project proposed by a full member and approved by CO-LaN Management Board. CO-LaN contracts a service provider to assist CAPE-OPEN component developers in efficient testing and debugging of CAPE-OPEN interfaces. If additional days are needed, this will be done through separate agreements between the developer and the consultant organisations. The service provider provides support directly to nominated third-party developers of CAPE-OPEN components, as agreed by CO-LaN. This scheme asks for consultancy providers to be approved by the CO-LaN. So a selection process was developed and took place.

\subsection{CO-LaN Approved Consultancy Services}

In order to select consultancy providers, CO-LaN issued a Request for Bids (RFB) on that topic as agreed for by the CO-LaN Management Board on October 30, 2003. The $\mathrm{CO}-\mathrm{LaN}$ frequently calls for bids on its testing software and other objects of interest. This specific RFB stated that CO-LaN wants to help independent developers of PMCs and PMEs to reach CAPE-OPEN compliance. For this purpose, CO-LaN is establishing a programme of CO-LaN Approved Consultancy Services by which organisations and companies with proven record of CAPE-OPEN technical expertise will be partially funded by CO-LaN to assist developers achieve compliance.

The typical task for a consultancy is to support a PMC or a PME developer to migrate its software by providing technical advice. Bidders had to demonstrate to CO-LaN their expertise in $\mathrm{CO}$ implementation as well as to demonstrate total commitment to CAPEOPEN in process modelling and simulation, i.e. by promoting CAPE-OPEN as their only method of integrating external components, where relevant.

Four consultancy providers have been selected and endorsed by the CO-LaN Management Board on December 19, 2003: Aspen Technology Inc. (AspenTech), JeanPierre Belaud from E.N.S.I.A.C.E.T., RSI, and Universita Politecnica de Catalunya and have been endorsed. The following specific approvals were issued:

- AspenTech (www.aspentech.com) has been approved for all aspects of the use of CAPE-OPEN Unit, Thermo and Common interfaces in COM software components. This results from their active involvement throughout the definition and implementation of CAPE-OPEN standards.

- Jean-Pierre Belaud has been approved for the use of CAPE-OPEN Unit, Solver and SMST interfaces in CAPE-OPEN-compliant software components, particularly in CORBA project applications. Advice on all $\mathrm{CO}$ interfaces and documentation is available. Access to students for assisting in project development is possible.

- RSI (www.rsi-france.com) has been approved for the use of CAPE-OPEN Unit and Thermo interfaces in both steady-state and dynamic CAPE-OPEN-compliant software components, particularly for real time COM applications.

- Universita Politecnica de Catalunya, Dpt. Enginyeria Química (www.eq.upc.es) has been approved as a consultant for the use of all CO major interfaces in CAPEOPEN-compliant software components, in both CORBA and COM applications. Access to small teams of students to assist in project development is possible. 
So the Consultancy Scheme was operational by the beginning of 2004. After presenting the scheme to the whole membership at the CO-LaN 2004 Annual Meeting on February 13,2004 , it was widely publicized in Volume 8 (July 2004) of the CAPE-OPEN Update Newsletter distributed electronically to the CAPE community. In June 2004 a first consultancy action took place.

\section{Application of the CAPE-OPEN Consultancy Scheme}

AixCAPE is an industrial consortium aiming at speeding up the use of new technologies in industrial practice (www.aixcape.org). BASF AG, BAYER Technology Services, Dow Chemical, Shell International Chemical, Degussa and ARKEMA are industrial members of this consortium. AixCAPE decided to migrate a shortcut algorithm tailored at non-ideal mixture separations and to make it available as a unit operation module. The following benefits were targeted:

- Simplified application of the algorithm. Where the academic prototype needed a lot of manual input for stream data, a CAPE-OPEN unit operation can easily get the same information from the Material Objects connected to its ports.

- Computation of complex separation sections can be performed easily since propagation of results through streams is handled by the PME.

- Consistent usage of thermodynamic models could be achieved.

\subsection{Problem definition}

Using the Consultancy Services Request form available from the CO-LaN web site, AixCAPE requested help to resolve two problems:

- The GUI for their Unit Operation did not get displayed in Aspen Plus although it worked in HYSYS and in the CAPE-OPEN tester.

- Simple property calculations such as fugacity coefficient did not work although they were able to access constant component properties (like molecular weight) and to calculate flashes.

The CO-LaN accepted AixCAPE request for help and designated AspenTech as the consultancy provider to be used. In order to investigate these issues, AixCAPE supplied AspenTech with builds and source code for the Unit Operation.

\subsection{Resolution}

By running the AixCAPE unit operation using debugging tools, AspenTech was able to discover that:

- A basis qualifier was being specified for properties to which it did not apply. This lead to a failure to retrieve property values.

- The array of property name strings being passed to CalcProp (and other) calls was not correctly initialised resulting in an empty first entry which caused Process Modelling Environments to return an error.

- When requesting a pressure - vapour fraction (PVF) flash, the unit operation was requesting temperature also to be calculated. This caused Process Modelling Environments to raise an exception because temperature is not a property that can be calculated additionally via the CAPE-OPEN CalcProp method in this case normally it is a result of the flash. 
With these issues corrected in the AixCAPE ShortCut Toolbox code, it was possible to execute simulations cleanly within Aspen Plus and HYSYS.Process Process Modelling Environments.

The GUI issue has not been resolved. It was demonstrated that the GUI can be displayed if the Unit Operation displays a Message Box before displaying the GUI form. From this it was concluded that there is no fundamental technical issue with displaying forms written using .NET. However, it does seem that displaying a Message Box performs some initialisation that is required to display the GUI form. AspenTech will follow its escalation process for Microsoft issues to see if this can be resolved.

AixCAPE are satisfied with the results achieved. AspenTech spent 12 hours working on that Consultancy Request.

\section{Conclusion}

CO-LaN responded to a CAPE community need by developed the CAPE-OPEN Consultancy Scheme. This is one of several actions aimed at accelerating the adoption of the CAPE-OPEN industry standard. This specific action has proved to be successful immediately. A second consultancy action is currently underway on a part of the SolidSim project at Technical University Hamburg-Harburg. Several other potential recipients of the same kind of consultancy effort have been listed by CO-LaN.

Should you be in a position to take advantage of this Consultancy Scheme, you should contact a Director on the CO-LaN Management Board to express your needs. Names and contact information for all Directors can be found on the CO-LaN web site.

\section{References}

Belaud J.P., M. Pons, K. Irons, W. Merk and P. Banks, 2003, Missions of the CAPE-OPEN Laboratories Network. Communication presented at FOCAPO 2003, Coral Springs, FL. In I. Grossmann and C.M. McDonald (Eds.), Fourth International Conference on Foundations of Computer-Aided Process Operations: A View to the Future Integration of R\&D, Manufacturing and the Global Supply Chain (pp. 527-530). Austin, TX: Computer Aids for Chemical Engineering Education (CACHE). ISBN-0965589110.

Benqlilou C., S. Bel, M. Gonzalez, M. Pons, R. Szczepanski, A. Espuna, L. Puigjaner, 2004, Towards the Standardization of Thermodynamic and Physical Properties Packages. Communication presented at ESCAPE 14, Lisbon, Portugal. In A. Barbosa-Povoa and H. Matos (Eds.), Proceedings of $14^{\text {th }}$ European Symposium on Computer-Aided Process Engineering [CD-Rom].

CO-LaN, 2003, CAPE-OPEN standard v1.0. In www.colan.org.

Pons, M., 2003, Industrial Implementations of the CAPE-OPEN standard. In S. Pierucci (Ed.), Selected papers of the sixth Italian conference on chemical and process engineering (AIDIC Conference Series, vol. 6, pp. 253-262). Milan, Italy: Reed Business Information S.p.A. ISBN 0390-2358. 